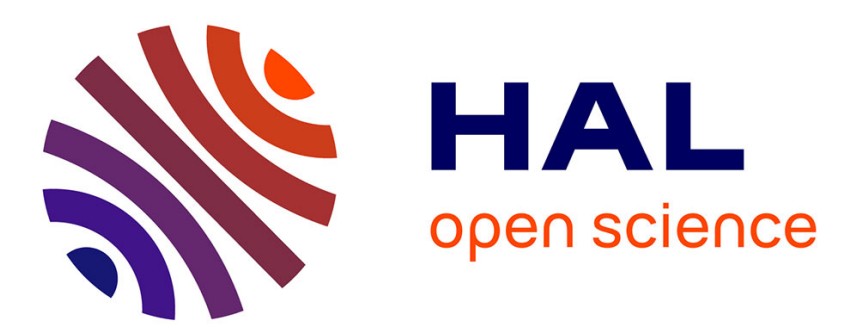

\title{
A Method to Determine the Maximum Side Perspective of Satellite with the Constraints of Mapping Accuracy
}

Jihong Yang, Haiwei Li, Yin Zhan, Liangshu Shi, Jinqiang Wang, Zhengchao

Chen

\section{- To cite this version:}

Jihong Yang, Haiwei Li, Yin Zhan, Liangshu Shi, Jinqiang Wang, et al.. A Method to Determine the Maximum Side Perspective of Satellite with the Constraints of Mapping Accuracy. 8th International Conference on Computer and Computing Technologies in Agriculture (CCTA), Sep 2014, Beijing, China. pp.13-22, 10.1007/978-3-319-19620-6_2 . hal-01420238

\section{HAL Id: hal-01420238 \\ https://hal.inria.fr/hal-01420238}

Submitted on 20 Dec 2016

HAL is a multi-disciplinary open access archive for the deposit and dissemination of scientific research documents, whether they are published or not. The documents may come from teaching and research institutions in France or abroad, or from public or private research centers.
L'archive ouverte pluridisciplinaire HAL, est destinée au dépôt et à la diffusion de documents scientifiques de niveau recherche, publiés ou non, émanant des établissements d'enseignement et de recherche français ou étrangers, des laboratoires publics ou privés.

\section{(c)(1)}

Distributed under a Creative Commons Attribution| 4.0 International License 


\title{
A Method to Determine the Maximum Side Perspective of Satellite with the Constraints of Mapping accuracy
}

\author{
Jihong Yang ${ }^{1}$, Haiwei $\mathrm{Li}^{2,4}$, Yin Zhan\&Liangshu $\mathrm{Shi}^{1}$, Jinqiang Wang ${ }^{3}$, \\ Zhengchao Chen ${ }^{4 *}$ \\ 1 Chinese Land Surveying and Planning Institute, Beijing 100035, China; \\ 2 School of Geosciences and Info-Physics, Central South University, Changsha \\ 410083, China; 3 Surveying and Mapping Bureau of Yunnan Province, Kunming \\ 650034, Yunnan, China; 4 Institute of Remote Sensing and Digital Earth, Chinese \\ Academy of Sciences, Beijing 100094, China \\ Corresponding author: Z.C.CHEN e-mail: zcchen@ceode.ac.cn
}

\begin{abstract}
In order to shorten the revisit period, and improve the efficiency of imaging, currently, all the in orbit high resolution remote sensing satellites adopt side-view imaging technology. When satellites imaging with a side perspective, it would inevitably cause the degradation of image quality, like spatial resolution reduction, image deformation incensement, and the reduction of positioning accuracy. In addition, with the increase of the side perspective, the error of DEM data will be amplified in the process of ortho-rectification, which, thus, will bring bigger error for image mapping accuracy. To address this problem, embarked from the impact of side view and DEM precision on image point error, this paper used Quick bird image data of the same area but different side perspectives, and simulate the impacts of different DEM elevation error on different side view image. At last we put forward a method to determine the maximum side perspective with map precision constraints, and set up a conversion relationship among satellite side view, DEM error and image point error. Finally, the paper combines the errors of 1:50000 DEM, ASTERGDEM and the requirement of ortho-image drawing, discusses and gives the values of maximum side perspective in the mountains and plains.
\end{abstract}

Keywords: DEM accuracy, satellite side perspective, ortho-rectification, geolocation accuracy

\section{Introduction}

Along with the continuous improvement of remote sensing satellites' spatial resolution, it also brought adverse effects of the image width and image acquisition efficiency reduction. In order to shorten the return cycle and to improve the efficiency of imaging, currently, on-orbit high resolution remote sensing satellites use the sideview imaging technology to improve the flexibility towards observation objects and image acquisition efficiency. As is well-known, when the satellites side-view imaging, 
the observation path is increased, the relative geometric relationship between satellite and ground objects is changed, which caused the degeneration of image quality ${ }^{[1]}$ (such as the spatial resolution reduced, image deformation increase, etc.) According to the principle of projection, from a point in space (viewpoint) observing the surface, each point on the surface along with topographic relief will generate geometric distortion on the image. The specific performance on the optical remote sensing imageries is as follows: the ground point, which is away from the viewpoint and with higher elevation, will fall to the opposite side of the viewpoint ${ }^{[2]}$. Ortho-rectification is to make use of terrain elevation model (DEM) to perform terrain distortion correction for each pixel in image, and make the image meet the orthographic projection, thus eliminating this distortion.

According to the error analysis of satellite imagery ortho-rectification model, the main factors affect the accuracy of ortho-rectification are as follows: orthorectification model error, satellite orbital attitude error, control point accuracy, DEM error, etc ${ }^{[3]}$. In which the ortho-rectification model commonly used strict physical model and rational polynomial model (RPC). Strict physical model are considered to be theoretically rigorous, thus, It can be considered without error; The RPC model is a simulation of strict physical model, the model conversion error is generally less than $1 \mathrm{~m}$ (Liu Shijie, 2008); The error of satellite orbit and attitude is decided by the control accuracy of the satellite itself and the in-orbit state, which cannot be changed by the average user. The precision of control points can be controlled through field measurement, currently using GPS can get higher precision. DEM data are input parameters of ortho-rectification, which generally directly adopt national fundamental geographic information data, the precision of is slightly different in the mountains and plains. In addition, when the satellites side-view imaging, their orbit and attitude control precision and measurement accuracy will decrease because of satellites or remote sensors' side swing ${ }^{[4]}$; Furthermore, according to the theory of projection, the increase of side perspective will definitely magnify the effects caused by DEM error. Therefore, the satellite side perspective is also one of the main error sources of satellite images ortho-rectification.

It can be seen from the above error analysis of ortho-rectification, in general case, the factors to be considered most by users are side perspective and DEM errors. Many scholars have carried out some research work about the effects on image quality degradation and ortho-rectification precision caused by side perspective, and the effects on ortho-rectification precision caused by DEM errors. Such as Han Wenli, Yuan Xiuxiao, and Wang Xuejun, etc. Han Wenli(2010) quantitatively analyzed the impact on ortho-rectification precision caused by side perspective in theory, and proved the validity of its conclusions through experiments. The conclusions indicate that with increment of the side perspective, the image ortho-rectification accuracy will gradually decline, and the decrease amplitude is quite obvious ${ }^{[3]}$. Taking into account changes of the side perspective, Yuan Xiuxiao et al., 2009, established the geometry model of high-resolution satellite remote sensing image processing, which improved the image positioning accuracy on the ground targets. It concluded that the change of 
the side perspective is a factor that cannot be neglected in the high-resolution satellite remote sensing image geometry processing model ${ }^{[5]}$. Wang Xuejun et al., 2008, selecting different DEM scales, conducted ortho-rectification to the data with different side perspectives acquired by SPOT5. It certified that when SPOT5 imaging, the side perspective is the key factor leading layer-over phenomenon of SPOT5 orthophoto products, with the premise of ensuring the accuracy of ground control points. It also certified that in the calibration process the improvement of DEM accuracy cannot effectively eliminate layer-over phenomenon of the mountains with complex terrain and large elevation drop. About the impact on image correcting accuracy caused by DEM data, Shi Yuhua (2007), using two different DEM data, calibrated the same scene image. The results show that the ortho-rectification accuracy is higher with small basic grid and high-precision DEM data ${ }^{[6]}$.

However, these studies only consider the impact of unilateral side perspective or DEM error on ortho-rectification precision, and failed to combine both. Actually it is a coupling effect between side perspective and DEM error: DEM error will be amplified with the increase of side perspective; Errors caused by side perspective will change along with the change DEM data. As the input conditions of orthorectification the accuracy of DEM data are often unable to change. Therefore, in practice, it is more significant to study how to determine the maximum satellite observation side perspective in the premise that there is some error in DEM data, as well as guarantee certain accuracy of ortho-rectification and mapping. Aiming at this problem, this paper adopted Quickbird image data with different side perspectives in Beijing, proposed a method to determine the maximum satellite imaging side perspective with certain constraints of mapping accuracy by simulating different influence law on the accuracy of image ortho-rectification caused by DEM elevation error, and established a quantitative conversion relationship among satellite side perspective, DEM error, and the error of ortho-rectification image points.

\section{Experiments and Methods}

\subsection{Experimental DATA}

The experimental area is the northern part of Beijing, including plain and mountain. Experimental data are multispectral image collected by using high-resolution Quickbird satellite in 2002 to 2012. With $2.44 \mathrm{~m}$ resolution, side perspective spanning from $5.6^{\circ}$ to $43.7^{\circ}$ ten different angles in total. Image side perspective spanned broadly, evenly distributed, specifically details can be seen in the table below.

Table 1 Test data tables side perspective Statistics

\begin{tabular}{l|l}
\hline Satellite Name & Side perspective $\left({ }^{\circ}\right)$ \\
\hline
\end{tabular}




\begin{tabular}{l|llllllllll} 
QuickBird & 5.6 & 10.4 & 14.4 & 16.3 & 22.1 & 28.2 & 31.8 & 34.3 & 38.9 & 43.7 \\
\hline
\end{tabular}

\subsection{Experimental Methods}

According to the principle of photogrammetry, the comparation between image point error caused by the side perspective and imaginary horizontal photograph as Figure 1(a) shows.

$P$ is the tilted photograph and $P 0$ is the imaginary horizontal photograph with the same observation conditions. They intersect at isocon line $h c$. Imagine that ground point $M$ is $(m)$ on tilted photograph $P$ and $\left(m_{0}\right)$ on horizontal photograph $P_{0}$, respectively. After two photograph overlapping along isocon line, the position of $(m)$ in the horizontal photograph is $m$, obviously $m m_{0}$ is the tilt error. With the increase of side perspective, the tilt error will become bigger. Image point error caused by the relief is shown as follows(right). To facilitate discussion, assume that photograph is horizontal, and the height difference between ground point A and the datum is $h$. The image point of ground piont $A$ is point $a$ on the photograph; the projection of $A$ on the datum is $A_{0}$, whose image point is $a_{0} . a a_{0}$ is the displacement of image points caused by the relief. As can be seen, the displacement of image points will increase with the increasement of height difference $h^{[7-9]}$.

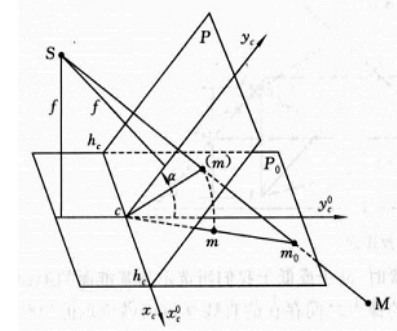

(a)

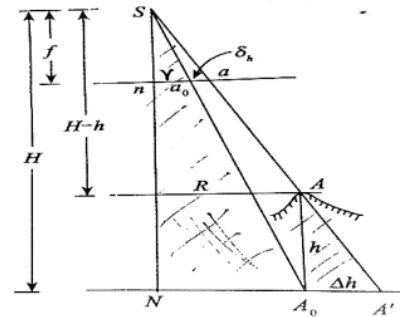

(b)

Figure 1 the error of displacement of image points caused by side perspective and relief((a): side perspective; (b): relief)

During the process of ortho-rectification, image points error will be affected by side perspective and relief at the same time. In the existing research results, different influence factors are often analyzed independently, which ignores the mutual influence and internal relations of different factors and is not real and comprehensive enough to reflect the objective situation of data. Because the strict physical model to calibrate the satellite image contains quantities of trigonometric functions and complex variables. If directly use strict physical model to deduce the error model of ortho-rectification, it will be very complicated. It is also why many scholars assume that the variables are independent in the derivation of the law of ortho-rectification error $^{[10][11]}$. In addition, RPC model is an approximate mathematical simulation of the physical model. RPC model without satellite orbit and attitude parameters, there is no way to establish the error model of ortho-rectification, DEM and orbit attitude parameters. Based on these considerations, this paper used the real images with 
different side perspectives and corresponding parameters, analyzed the relationship among side perspective, DEM accuracy, and ortho-rectification precision, when inputting different DEM elevation data, and derived the function among variables based on experimental data statistics. The specific process is shown in figure 2 .

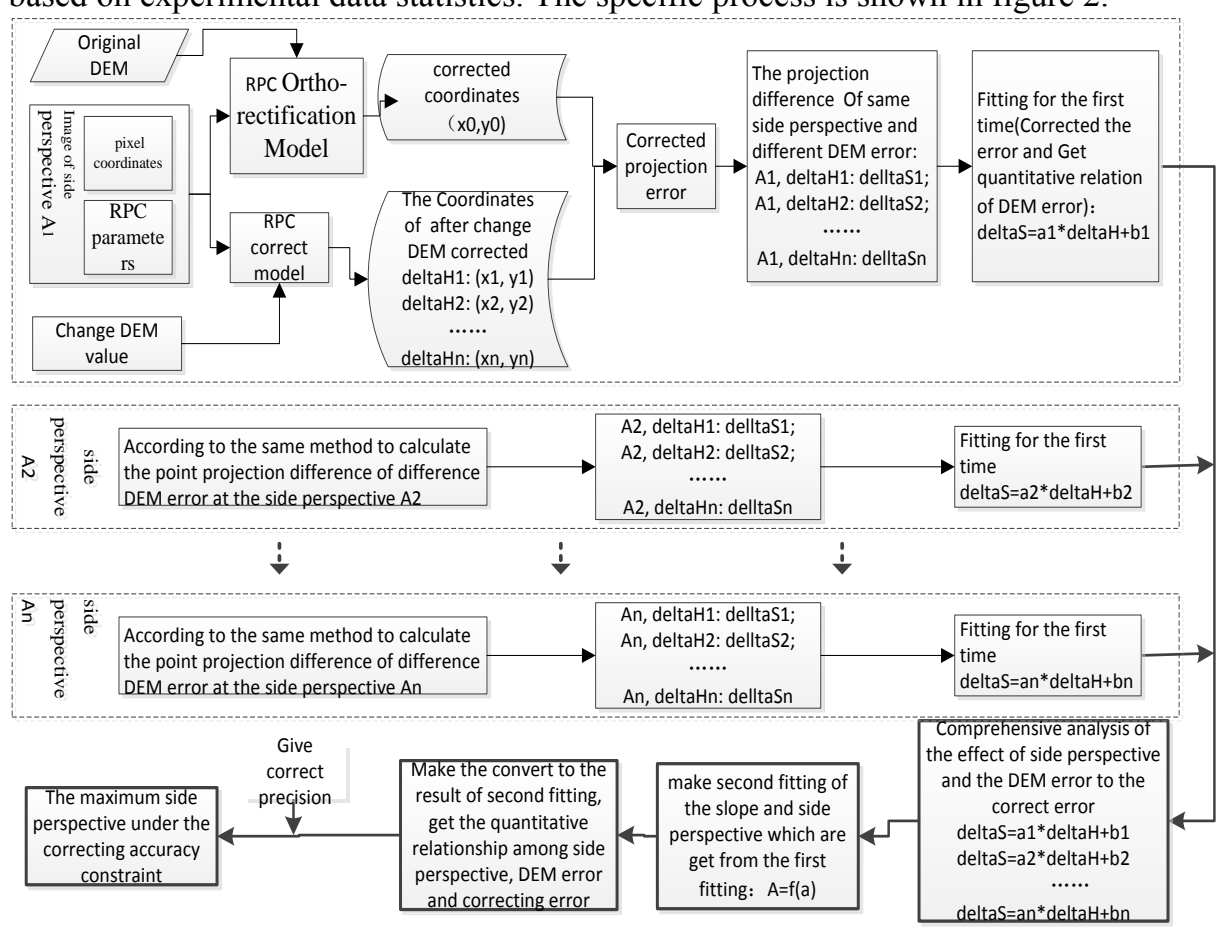

Figure 2 the analysis process of the effects on ortho-rectification precision caused by side perspective and DEM error

According to the figure 2, the concrete steps and methods are as follows:

Input the satellite images and their corresponding parameters, original DEM data into RPC model, when the side perspective is given. Using RPC model, conduct ortho-rectification against specified pixel. Record the calibrated coordinates as the initial value of the corrected geographical coordinates.

Change the DEM elevation value without other parameters changed. Conduct ortho-rectification against specified pixel by using RPC ortho-rectification model, then Obtain the corrected value of geographical coordinates which is derived from formula (1). The displacement of the coordinates in step (1) is also calculated by formula (1). Given the different DEM elevation values, obtain the ortho-rectification error( one column in Table 2)with the same side perspective and different DEM elevation error.

$$
\delta S=\sqrt{(\Delta x)^{2}+(\Delta y)^{2}}
$$




\section{Results and Discussion}

Table 2 the correcting error(m) at different side perspective and DEM error of QuickBird

\begin{tabular}{|c|c|c|c|c|c|c|c|c|c|c|}
\hline $\begin{array}{c}\text { Ang } \\
\text { DEM error }\end{array}$ & $\mathbf{5 . 6}^{\circ}$ & $\begin{array}{c}\mathbf{1 0 . 4} \\
\circ\end{array}$ & $\begin{array}{c}\mathbf{1 4 . 4} \\
\circ\end{array}$ & $\begin{array}{c}\mathbf{1 6 . 3} \\
\circ\end{array}$ & $\begin{array}{c}\mathbf{2 2 . 1} \\
0\end{array}$ & $\begin{array}{c}\mathbf{2 8 . 2} \\
\circ\end{array}$ & $\begin{array}{c}\mathbf{3 1 . 4} \\
\circ\end{array}$ & $\begin{array}{c}\mathbf{3 4 . 3} \\
\circ\end{array}$ & $\begin{array}{c}\mathbf{3 8 . 9} \\
\circ\end{array}$ & $\begin{array}{c}\mathbf{4 3 . 7} \\
\circ\end{array}$ \\
\hline $\mathbf{2 0 m}$ & 2.080815 & 4.101156 & 5.566553 & 6.112367 & 9.033995 & 11.74509 & 13.44905 & 14.92516 & 17.84973 & 22.63661 \\
\hline $\mathbf{4 0 m}$ & 4.162622 & 8.202106 & 11.13377 & 12.22391 & 18.0683 & 23.49034 & 26.89715 & 29.84912 & 35.70007 & 45.27226 \\
\hline $\mathbf{6 0 m}$ & 6.24443 & 12.30424 & 16.70032 & 18.33628 & 27.10355 & 35.23445 & 40.3462 & 44.77428 & 53.54963 & 67.9079 \\
\hline $\mathbf{8 0 m}$ & 8.325118 & 16.4054 & 22.26658 & 24.44782 & 36.13786 & 46.97954 & 53.79524 & 59.69846 & 71.39936 & 90.54354 \\
\hline $\begin{array}{c}\mathbf{1 0 0} \\
\mathbf{m}\end{array}$ & 10.40693 & 20.50635 & 27.83285 & 30.55962 & 45.17216 & 58.72381 & 67.24335 & 74.62242 & 89.24892 & 113.1792 \\
\hline $\begin{array}{c}\mathbf{1 2 0} \\
\mathbf{m}\end{array}$ & 12.48873 & 24.6075 & 33.3994 & 36.67116 & 54.20646 & 70.4689 & 80.69239 & 89.5466 & 107.0979 & 135.8148 \\
\hline $\begin{array}{c}\mathbf{1 4 0} \\
\mathbf{m}\end{array}$ & 14.56955 & 28.70964 & 38.96566 & 42.78353 & 63.24076 & 82.21301 & 94.1405 & 104.4708 & 124.9468 & 158.4495 \\
\hline $\begin{array}{c}\mathbf{1 6 0} \\
\mathbf{m}\end{array}$ & 16.65135 & 32.81059 & 44.53192 & 48.89507 & 72.27507 & 93.95727 & 107.5895 & 119.3947 & 142.7957 & 181.0839 \\
\hline $\begin{array}{c}\mathbf{1 8 0} \\
\mathbf{m}\end{array}$ & 18.73204 & 36.91174 & 50.09847 & 55.00687 & 81.30906 & 105.7014 & 121.0376 & 134.3189 & 160.6447 & 203.7186 \\
\hline $\begin{array}{c}\mathbf{2 0 0} \\
\mathbf{m}\end{array}$ & 20.81385 & 41.01269 & 55.66474 & 61.11842 & 90.34337 & 117.4465 & 134.4854 & 149.2429 & 178.4936 & 226.3533 \\
\hline
\end{tabular}

Figure 3 shows that using experimental data with different side perspectives, the paper calculated the influence law distribution on ortho-rectification accuracy caused by DEM error. From the numerical distribution and the fitting results in figure 3 , it can be seen that under the same side perspective, pixel positioning errors increase with the increasement of DEM elevation values; the larger the side perspective is, the greater the pixel positioning is. And the pixel positioning is more obviously affected by DEM values. The change is completely linear relationship, and fitting precision $\mathrm{R}^{2}$ is 1 . For instance, with the side perspective $5.6^{\circ}$, the positioning error is $2.08 \mathrm{~m}$, when DEM error is $20 \mathrm{~m}$. While it increases to $20.8 \mathrm{~m}$, when DEM is $200 \mathrm{~m}$, which increased 10 times. Figure 4 shows the impact on ortho-rectification accuracy caused by side perspectives with certain elevation error. It can be seen that under certain DEM elevation error, the image positioning error will be bigger with larger side perspectives. After fitting, the variation can be expressed by a quadratic polynomial. Fitting accuracy $\mathrm{R}^{2}$ is almost equal to 1 , like, when the elevation error is $20 \mathrm{~m}, \mathrm{R}^{2}=0.9996$. 


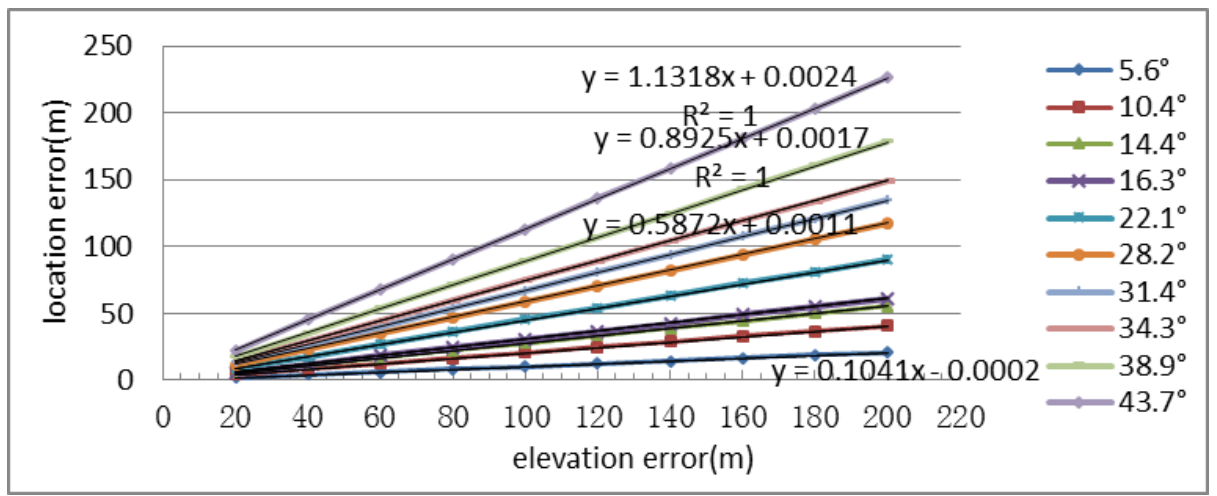

Figure 3 the effect of elevation error to the position error at different side perspective

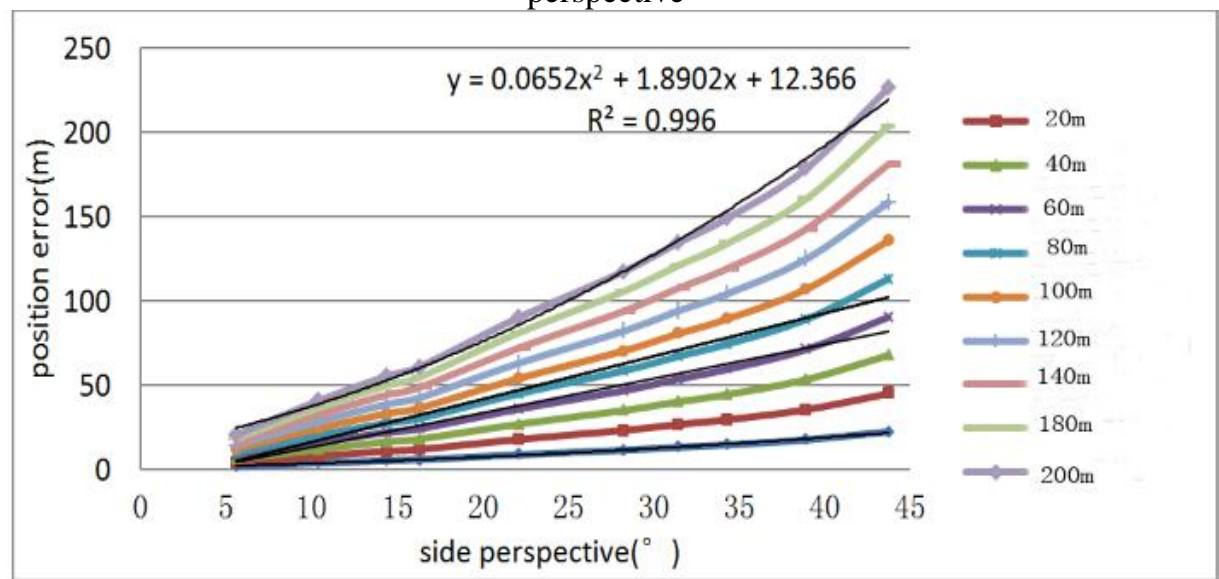

Figure 4 the effect of side perspective to the position error at different elevation error

Comprehensively analyze the impact on ortho-rectification accuracy caused by side perspective and DEM error. Table 2 and Figure 3, Figure 4, various variations combine the impact on image points displacement caused by DEM elevation values and side perspective. They reflect the variations and quantitative relationship of the three variables.

According to figure 3 , it can be seen that with certain side perspective, the impact relationship on ortho-rectification accuracy caused by DEM error is completely linear, and the slope of the fitting line increases with the increasement of side perspectives.

In order to investigate the relationship between the fitting line slope and side perspective, the slope and intercept of all the test data are presented in table 3. As can be seen from the table, the slope of the fitted line increases with the increasement of side perspectives, but the intercepts are relatively small. The Maximum of intercept is no more than $0.0024 \mathrm{~cm}$, which can be ignored as infinitesimal in practical applications. In order to further give the quantitative relationship of fitting line slope and side perspective, Figure 5 shows the slope distribution curve of the fitting line 
along with the change of side perspective and fitting results of experimental data in this paper.

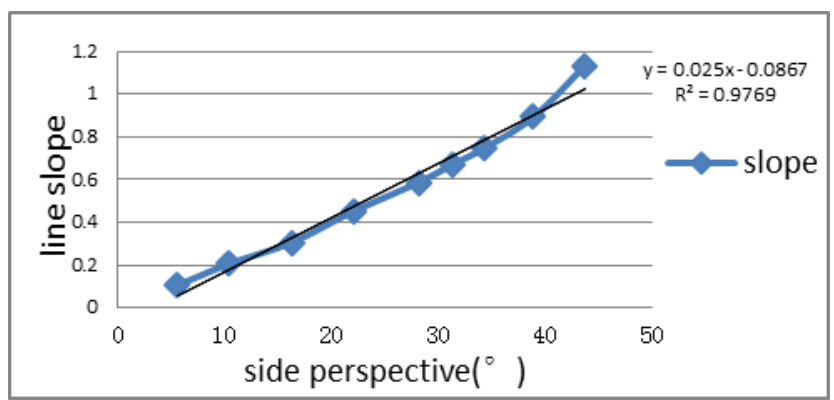

Figure 5 the relationship of side perspective and line slope

Table 3 the slope and intercept of fitting straight line between DEM error and correcting precision with different side perspectives

\begin{tabular}{|c|c|c|c|c|c|c|c|c|c|c|}
\hline $\begin{array}{c}\text { Side } \\
\text { perspective } \\
\left({ }^{\circ}\right)\end{array}$ & 5.6 & 10.4 & 14.4 & 16.3 & 22.1 & 28.2 & 31.4 & 34.3 & 38.9 & 43.7 \\
\hline Slope & 0.1041 & 0.2051 & 0.2783 & 0.3056 & 0.4517 & 0.5872 & 0.6724 & 0.7462 & 0.8925 & 1.1318 \\
\hline $\begin{array}{l}\text { Intercept } \\
(\mathrm{m})\end{array}$ & $\begin{array}{c}- \\
0.0002\end{array}$ & $\begin{array}{c}- \\
0.00009\end{array}$ & 0.0007 & 0.0006 & 0.00005 & 0.0011 & 0.0006 & 0.0013 & 0.0017 & 0.0024 \\
\hline
\end{tabular}

From numerical distribution and fitting results in the figure 5, it can be seen that it is also a linear relationship between the side perspective and fitting line slope. Fitting accuracy R2 reached 0.9769 . Therefore, the relationship between fitting line slope of DEM error and ortho-rectification and satellite side perspective can be considered linear. (Eq. 2):

$$
\text { slope }=a \times \text { angle }+b
$$

In which slope is the slope of the fitting line; angle is the side perspective; $a, b$ is coefficient of line fitting. According to the physical meaning, combining Figure 3 and Equation 2, slope y is the ratio of correcting error and elevation error. Namely:

$$
\text { slope }=\delta S / \delta H
$$

Where $\delta S$ is correcting error; $\delta H$ is DEM error.

Equation 4 can be derived from combining Equations 2 and 3:

$$
\delta S / \delta H=a \times \text { angle }+b
$$

Equation 4 describes the approximate relationship among side perspective, DEM error and error of pixel correcting.

Using the experimental data in this paper, it can derive that a equals to 0.025 , and $b$ equals to -0.0867 . Because $b$ is less than $9 \mathrm{~cm}$, which can be regarded as infinitesimal and ignored. The simplified function among side perspective, DEM error and pixel 
correcting accuracy can be derived from inputting the formula 4 with $\mathrm{a}=0.025$ and discarding $b$.

$$
\text { angle }=40 \delta S / \delta H
$$

Equation 5 is a special case of 4 . When the precision requirements of positioning error, DEM data accuracy are known, the maximum side perspective of QuickBird satellite can be caculated, which meet the accuracy requirements.

\section{APPLICATION}

This paper, by using QuickBird satellite images in Beijing, deduced the effects on the precision of image ortho-rectification caused by side perspective and DEM error, and provided the quantitative functional relationship among the three. With this function, if given the actuary request of image ortho-rectification and DEM accuracy, it is possible to determine the largest satellite imaging side perspective. Thus, before collecting data, it can help avoid the potential trouble of ortho-rectification accuracy overrunning in later phase.

Based on the Quickbird test data statistics model, this paper, using the 1:50 000 DEM of China and the American ASTER GDEM to perform ortho-rectification, respectively calculated the maximum allowable side perspective on the mountains and plains.

According to the requirements of GBT 13977-1992 "1:5000,1:10000 topographic maps and aerial photogrammetry field work norm", plane position mean error of feature points on the map is less than $0.5 \mathrm{~mm}$ on plains and hilly lands, $0.75 \mathrm{~mm}$ on mountainous and alpine areas.

Corresponding 1:10000 topographic mapping requirements, the error of image calibration is no more than $5 \mathrm{~m}$ on plains and hilly lands, $7.5 \mathrm{~m}$ on mountainous and alpine areas.

In addition, the grid size of our nationwide 1:50000DEM $25 \times 25 \mathrm{~m}$ is known. The elevation mean error of grid points comparing to the near field elevation control point is not more than $4 \mathrm{~m}$ on plains, not more than $11 \mathrm{~m}$ on mountains[1]. The elevation accuracy of American ASTER GDEM is 20m(ASTER Global DEM Validation Summary Report. 2009).

1) According to formula (5), When the national 1:50000 DEM is used of $\delta H$ plains is $4 \mathrm{~m}$, and $\delta S$ is $5 \mathrm{~m}$. Then in order to meet the mapping requirements of 1:10000, the side perspective on the plain areas of Quickbird image must be less than or equal to $50^{\circ}$. $\delta H$ of mountains is $11 \mathrm{~m}$, and $\delta S$ is $7.5 \mathrm{~m}$, the corresponding side perspective must be less than or equal to $27.3^{\circ}$.

2)when American ASTER GDEM is used, $\delta H$ of plains and mountains is $20 \mathrm{~m}$, in order to meet the mapping requirements of 1:10000, the error of image calibration is no more than $5 \mathrm{~m}$ on plains and hilly lands and $7.5 \mathrm{~m}$ on mountainous and alpine areas. Taking elevation value $5 \mathrm{~m}$ into computation, the imaging side perspective of Quickbird should be less than or equal to $10^{\circ}$ 。 
Note that the formula (6)is deduced based on the results of data regression statistics in Beijing experimental zone. Due to the limitations of statistical samples and terrain differences, these results are only for reference.

In addition, given the current main on-orbit high-resolution commercial satellites, including QuickBird, WordView, IKONOS, etc. Due to the similarity of Orthorectification error and solutions, the method should also be applicable to other similar type of satellite platforms.

\section{Conclusions}

This paper established the quantitative conversion relationship among satellite side perspective, DEM error, ortho-rectification, and image rectification, proposed a method to determine the maximum imaging side perspective with map precision constraints, and provided guidance on data selection and processing. When known DEM data accuracy and the accuracy that ortho-rectification expected to achieve, the maximum side perspective of the satellite can be calculated to meet those conditions. On the other hand, when the existing satellite data side perspective is known, the minimum accuracy of DEM data can be calculated to achieve the expected orthorectification precision. Because the model in this article is obtained by the analysis of real data, thus, the authenticity of the results is guaranteed, and the conclusion is also in line with the actual situation. In addition, the ideas and methods adopted by this paper are also applicable to the analysis and study of other sensors with the same kind.

\section{Acknowledgment}

In this paper, the test data are provided by Beijing Aerospace Shi Jing Information Technology Co, Ltd.

\section{References}

[1] GBT_13977-1992. 1:5000, 1:10000 topographic maps and aerial photogrammetry field work norms [D].

[2] Yong W, Xue H. Remote Sensing Refined Explanation [M]. Surveying and Mapping Press, 1993.

[3] Han WL. Research on the Influence of Correcting Precision Caused by Satellite Incidence Angle [J]. Beijing Surveying and Mapping, 2010, 04): 20-2.

[4] He HY, Wu CD, Wang XY. Study of Influence of Swinging on the Systemic Parameters of the Satellite and CCD Camera [J]. Spacecraft Recovery and Remote Sensing, 2003, 04): 14-8.

[5] Yuan XX, Cao JS, Yao N. A Rigorous Geometric Model Considering the Variety of Side Watch Angle for High-resolution Satellite Imagery [J]. Acta Geodaeticaet Carto graphica Sinica, 2009, 02): 120-4. 
[6] Shi YH. Research of Quickbird Image on Orthocorrection and Updating of Relief Map [J]. Geospatial Information, 2007, 06): 45-7.

[7] Zhang JQ. Photogrammetry [M]. Wuhan: Wuhan University Press, 1995.

[8] Okamoto A, Akamatu S, Hasegawa H. Orientation Theory for Satellite CCD LineScanner Imageries of Mountainous Terrains [J]. International Archives of Photogrammetry and Remote Sensing, 1993, 29(205-.

[9] Okamoto A. Orientation theory of CCD line-scanner images [J]. International Archives of Photogrammetry and Remote Sensing, 1988, 27(B3): 609-17.

[10] Yan-wei(1)(2) R, Xin-ming(2) T, Hua-bin(2) W, et al. The SPOT HRG images orthorectification experiment and precision analysis without GCPs [J] [J]. Science of Surveying and Mapping, 2008, 3(029.

[11] ZHANG Li-ping1,CUI Yong-li2,WANG Bao-shan2(1.The Third Heilongjiang Surveying and Mapping Engineering Institute,Harbin 150086,China;2.The Second Heilongjiang Surveying and Mapping Engineering Institute,Harbin 150086, China);Analysis on the Accuracy of Quick Bird Image Ortho-Rectification under Different Terrain Condition[J];Geomatics \& Spatial Information Technology;2009-04 